Relations industrielles

Industrial Relations

\title{
Dignity at Work par Randy HoDson, New York : Cambridge \\ University Press, 2001, 320 p., ISBN : 0-521-77131-5 et \\ 0-521-77812-5.
}

\section{Jacques Bélanger}

Volume 58, numéro 1, hiver 2003

URI : https://id.erudit.org/iderudit/007378ar

DOI : https://doi.org/10.7202/007378ar

Aller au sommaire du numéro

Éditeur(s)

Département des relations industrielles de l'Université Laval

ISSN

0034-379X (imprimé)

1703-8138 (numérique)

Découvrir la revue

Citer ce compte rendu

Bélanger, J. (2003). Compte rendu de [Dignity at Work par Randy Hodson, New York: Cambridge University Press, 2001, 320 p., ISBN : 0-521-77131-5 et 0-521-77812-5.] Relations industrielles / Industrial Relations, 58(1), 154-157. https://doi.org/10.7202/007378ar

Tous droits réservés (C Département des relations industrielles de l'Université Laval, 2003
Ce document est protégé par la loi sur le droit d'auteur. L’utilisation des services d’Érudit (y compris la reproduction) est assujettie à sa politique d'utilisation que vous pouvez consulter en ligne.

https://apropos.erudit.org/fr/usagers/politique-dutilisation/ 
market in 1938, 3.5 million were excluded from coverage under the SSA because farm workers and domestic workers were excluded from coverage (p. 148). The exclusion of domestic workers had a particularly sharp effect on women who worked in factories during World War II. Many of these women returned from covered factory jobs to uncovered domestic service jobs having paid into the social security system, but having not achieved enough quarters (at least 10 years' worth) to collect benefits based on their contributions.

Chapter Four talks about the development of federal taxation policy in the mid-1900's and how taxation policy affected women. One interesting thread in the gendered debate was the role that congressional representatives from community property states (most of which were states that became U.S. territory after the U.S.-Mexico war which ended with the Treaty of Hidalgo in 1848) played. In community property states, wives own equally the married couple's property including income earned during the marriage (p. 176). Chapters Five and Six discuss the development of federal legislation governing equal pay for equal work for men and women and other civil rights laws.

In Pursuit of Equity is a complex and interesting exposition on the way policymakers' gendered views affected policy debates and outcomes. It shows the way systemic discrimination seeped into almost all of the legislation that governs the workplace to this dayfrom tax policy to unemployment insurance to the establishment of a national minimum wage. The book is also a cautionary tale for this century's policymakers. Human beings may never be able to see beyond the cultures and ideologies that formed us. Nevertheless, when we set ourselves to the task of designing laws and institutions that affect working people's lives, we must take extra care to avoid systematizing our prejudices into those laws and institutions.
Tequila J. BROOKS

Commission for Labor Cooperation Secretariat Washington, DC

\section{Dignity at Work \\ par Randy Hodson, New York : Cambridge University Press, 2001, 320 p., ISBN : 0-521-77131-5 et 0-521-77812-5.}

À partir d'une étude systématique des connaissances accumulées au fil des ans à travers les monographies d'entreprises, Randy Hodson construit un cadre interprétatif autour du concept de dignité au travail.

Suivant une démarche méthodologique rigoureuse, et bien exposée dans l'ouvrage, l'auteur a procédé à un repérage exhaustif des études ethnographiques publiées en langue anglaise. Après avoir identifié 365 ouvrages, l'auteur et son équipe en ont retenu 86 pour l'ana- lyse quantitative, à partir d'un système de codification élaboré. Les monographies retenues sont celles qui résultent de plus de six mois d'observation dans une même organisation et présentent des informations complètes sur un groupe occupationnel donné (ou parfois deux, ce qui permet une analyse sur 108 cas). Ces critères excluent des monographies que bien des spécialistes considèrent comme marquantes, et je pense par exemple à celle de Batstone, Boraston et Frenkel (1977) ou encore celle plus récente de Barker (1999) sur les équi- 
pes de travail. Mais cette remarque n'est pas fondamentale : l'ajout de tel ou tel ouvrage n'aurait changé ni les analyses statistiques ni l'argumentation, d'autant plus que la discussion s'appuie sur une bibliographie beaucoup plus large que les seules études retenues pour l'analyse quantitative. Soulignons toutefois une limite inévitable, la grande majorité des études retenues ont été réalisées aux États-Unis ou en Grande-Bretagne, alors que les quelques monographies portant sur la France, où cette tradition ethnographique fut longtemps si riche, sont celles traduites en langue anglaise (M. Crozier et R. Linhart). L'auteur insiste (p. 56-57), il ne s'agit pas d'une métaanalyse, qui consiste à faire le bilan des études quantitatives sur tel effet spécifique, mais bien d'une étude quantitative réalisée à partir d'études ethnographiques.

Utilisé ici dans un sens fondamental, avec notamment la connotation de respect de soi et de respect des autres, le concept de dignité est riche et prometteur. Il est opérationnalisé autour de quatre entraves ou menaces à la dignité au travail (la mauvaise gestion, la surcharge de travail, les limites à l'autonomie, et les contradictions suscitées par l'implication des employés) et de quatre possibilités d'action pour sauvegarder la dignité (la résistance, la citoyenneté au travail, la recherche de sens, et les relations de groupe). Ces dimensions, sur deux axes, structurent l'ouvrage.

L'auteur n'a pas l'ambition de présenter un cadre explicatif général. Notamment, il n'y a pas de correspondance entre les quatre menaces et les quatre possibilités d'action, ces dernières constituant plutôt un éventail de solutions possibles pour pallier les obstacles à la dignité. Aussi, me semble-t-il, la signification des notions d'autonomie et d'implication des employés pose une certaine difficulté dans le modèle conceptuel suggéré. Ces limites n'invalident pas pour autant le concept de dignité au travail, qui sera retenu à juste titre par bien des lecteurs, mais suggèrent une poursuite de la réflexion théorique.

Pour chacune des menaces et des contraintes à la dignité au travail, l'auteur présente une analyse statistique selon plusieurs indicateurs, en faisant le plus souvent porter la discussion sur les facteurs qui atténuent ou amplifient celles-ci. Parmi ces facteurs, les différentes formes de résistance et de citoyenneté au travail (engagement, fierté à l'égard de son travail, satisfaction, etc.) retiennent suffisamment l'attention pour constituer des thèmes récurrents. Ainsi, le chapitre 4 démontre que « mismanagement and abuse have a central role in generating resistance and undermining citizenship in the workplace » (p. 83). Bien que la discussion à partir de l'analyse statistique devienne parfois assez technique, le texte est à la fois plus agréable et plus convaincant parce qu'il est illustré de nombreuses citations tirées des monographies.

Au fil des chapitres d'analyse, puis de façon plus explicite dans la dernière partie de l'ouvrage, le fil conducteur de l'ouvrage devient évident : le principal obstacle à la dignité au travail se situe du côté du management. De façon récurrente, l'analyse de ces monographies montre que les abus de la part du management et la mauvaise gestion suscitent la résistance des employés et réduisent l'engagement au travail et l'efficience de façon plus générale. De fait, le chapitre 8 rappelle aussi que, loin de favoriser la solidarité parmi les salariés, la mauvaise gestion suscite des tensions et conflits parmi ceux-ci. Ainsi, « solidarity among coworkers is higher under conditions of competent management than under mismanagement » (p. 217).

En revanche, la possibilité d'appliquer son savoir-faire et sa créativité, ainsi que la fierté de bien faire son travail constituent la principale source de bien-être au travail. Ce faisant, Hodson met en lumière l'un des traits marquants de la sociologie du travail, à savoir 
«l'intérêt » des salariés à l'égard de l'efficience organisationnelle. La formule qui suit mérite d'être citée plus longuement parce qu'elle indique les implications plus larges de cette réflexion sur la relation d'emploi, réfutant du même coup des analyses encore trop récurrentes en relations industrielles, des analyses qui consistent à associer trop facilement l'efficience aux intérêts de l'employeur ou encore au management. "The efficiency of the production process and the quality of the product are only secondary goals for management - they are means to the end, which is profit... Workers have a different orientation. They have an abiding interest in the efficiency of production and in the quality of the product because their own well-being and the quality of their daily lives at work depend on efficiency and quality. Increasing the efficiency of production and the quality of the product is their principal, and often sole, avenue for ensuring organizational success and the continuation of their own jobs » (p. 256-257).

L'ouvrage cherche aussi à évaluer dans quelle mesure la participation et l'engagement des salariés favorisent ou non la dignité au travail. Les résultats, nuancés, sont dans l'ensemble positifs. Notamment, la proposition souvent avancée selon laquelle les équipes de travail atténuent la solidarité et suscitent un glissement de la loyauté, du collectif des salariés vers l'employeur, n'est pas appuyée par l'analyse des monographies (p. 187-189, 226). L'auteur souligne d'ailleurs en conclusion, sur une note positive, que les systèmes de production complexes et fondés sur les connaissances dépendront de plus en plus de l'engagement des salariés et de leur volonté d'utiliser leur savoir et leur expertise. «Working with dignity will not come automatically with new skills, but workers today, to a greater extent than at any time in history, have important new tools they can use in their struggle for dignity at work » (p. 269).
En puisant dans la richesse de ces monographies, la démarche quantitative de Hodson ajoute aux connaissances sur les rapports sociaux au travail. Elle met en valeur la contribution des études ethnographiques. Une limite importante de cet ouvrage, toutefois, a trait au traitement des institutions, un aspect central dans le champ des relations industrielles. Certes, le fait d'être syndiqué ou non et la distinction entre États-Unis et Grande-Bretagne sont parmi les variables considérées dans l'analyse quantitative. Mais pour le reste, le contexte de l'observation, pour ces monographies publiées entre 1952 et 1992, et la façon dont les formes institutionnelles conditionnent l'action ne transparaissent pas dans la discussion et l'analyse. Or, la possibilité de situer les rapports sociaux dans leur contexte, dans le temps et dans l'espace, et de porter attention à la culture et aux institutions, représente pourtant l'une des richesses de l'approche ethnographique. En somme, cet ouvrage incite à poursuivre dans la voie de l'observation en profondeur dans les milieux de travail.

Sur le plan théorique, le principal apport de cet ouvrage a trait à la réflexion sur la question classique de la structure et de l'action (surtout les pages 238-241 et 264-267). L'auteur a bien raison, « in order to understand workplace behaviors we need a theoretical model of the worker that is neither anesthetized nor limited to resisting management strategies of control » (p. 266). Dans l'ensemble du livre, l'accent porte beaucoup sur les moyens et les ressources par lesquels les salariés, de différentes catégories occupationnelles, peuvent souvent créer la cohésion sociale nécessaire pour résister ou dire non, et donner un sens à leur travail, malgré les contraintes associées à la relation d'emploi. L'espace social et l'autonomie qui contribuent souvent à réduire l'aliénation et à accroître la dignité au travail sont explorés avec beaucoup de pertinence. 
Dans les courants de recherche internationaux sur l'évolution du travail, Dignity at Work sera considéré comme un ouvrage de référence. Et pour les spécialistes de la sociologie du travail et de l'entreprise, son étude est incontournable.

\section{JACQUES BÉLANGER}

Université Laval

\section{Psychologie du travail et comportement organisationnel ( $2^{e}$ édition)}

par Shimon L. Dolan, Éric Gosselin, Jules CARRIÈre et Gérald LAMOUREuX, Montréal : Gaëtan Morin éditeur, 508 p., ISBN : 2-89105-803-8.

À la lumière des multiples transformations organisationnelles, les auteurs poursuivent l'objectif d'adapter les notions en psychologie du travail et en comportement organisationnel à la nouvelle réalité du travail.

Plusieurs orientations ont guidé la préparation de la seconde édition du volume. Premièrement, une orientation analytique : le livre offre un cadre d'analyse très structuré pour aider à résoudre les problèmes humains en milieu de travail. Les principes de la psychologie du travail et du comportement organisationnel sont abondamment illustrés à l'aide d'exemples d'entreprises. Deuxièmement, une orientation pédagogique : les cas ont été rédigés par une multitude de professeurs universitaires qui oeuvrent dans les secteurs de la psychologie du travail ou du comportement organisationnel. Les auteurs ont conservé les grilles d'autoévaluation de l'édition de 1996 afin de permettre aux étudiants de bien saisir la portée et l'incidence des concepts sur ses activités quotidiennes. Les auteurs ont malheureusement renoncé à joindre les allocutions « propos de chercheur renommé » et «point de vue d'un gestionnaire » de l'édition de 1996 à l'édition de 2002. Ces allocutions permettaient d'effectuer un pont entre la théorie et la pratique. Troisièmement, une orientation technologique : Dolan, Gosselin, Carrière et Lamoureux ont élaboré un cd-rom qui soumet des solutions pratiques et exhaustives aux cas du volume. Le cd-rom offre également une présentation Power Point détaillée du plan de cours d'introduction à la psychologie du travail et au comportement organisationnel. Le matériel technologique propose une variété de figures, de tableaux et de graphiques visuellement attrayants.

Sur le plan du format, le volume est très bien rédigé. Les auteurs adoptent un style de rédaction simple et direct. La limpidité et la cohérence de la structure du livre permettent de comprendre sans difficulté le cheminement conceptuel des auteurs. Les tableaux, les figures et les caricatures sont très compréhensibles afin de permettre la clarification des concepts. La vulgarisation des notions complexes constitue vraisemblablement la principale qualité pédagogique du livre.

Les deux premiers chapitres expliquent les fondements de la psychologie du travail et du comportement organisationnel. Les diverses écoles de pensée en psychologie (e.g., le béhaviorisme, la psychologie cognitive) et les modèles traditionnels en comportement organisationnel (e.g., le modèle mécanisme ; le modèle organique ; le modèle de contingence) sont revus en détail. Les auteurs ne décrivent pas suffisamment leur modèle contemporain du comportement organisationnel (p. 25). Il faut souligner l'effort important des auteurs pour retracer l'évolution des divers courants théoriques en psychologie du travail et en comportement organisationnel à l'aide du tableau 1.8 (p. 26-27). Cependant, ils n'ont pas défini sommairement les 